\title{
Improving perioperative outcomes in low-resource countries: It can't be fixed without data
}

\author{
Thomas G. Weiser, MD, MPH (D) Emmanuel M. Makasa, MBChB, MPH • Adrian W. Gelb, MBChB
}

Received: 16 July 2015/Revised: 23 July 2015/Accepted: 1 September 2015/Published online: 21 September 2015

(c) Canadian Anesthesiologists' Society 2015

It is a basic tenet of medicine to measure the effects of care. This includes not just immediate responses to treatments but also outcomes, the most dramatic and conclusive being mortality. Understanding death rates is essential for understanding the impact of perioperative care. It provides indispensable guidance for clinicians to determine the risks and benefits of intervention, for patients to weigh those risks, and for systems of healthcare to evaluate performance, learn from those that perform well (or poorly), and establish benchmarks for improvement.

In this issue of the Journal, Lillie et al. present an audit of 18,010 patients who underwent surgery at the University Teaching Hospital (UTH) in Lusaka, Zambia during 2012 a similar audit was reported 25 years previously. The authors reviewed the six-day postoperative mortality rates as well as the likelihood that the death was preventable. ${ }^{1}$ Of this total obtained from hospital administrative records, the investigators identified 11,688 surgical cases from operating room registers (a 35\% loss from the administrative $\log$ ). By cross-referencing data from the hospital mortuary as well as death reports from the surgical and obstetrics wards, the authors were able to identify 114 deaths that occurred within six days of surgery. Tellingly,

T. G. Weiser, MD, MPH ( $\square)$

Department of Surgery, Stanford University Medical Center, 300

Pasteur Dr, S067, Stanford, CA 94305, USA

e-mail: tweiser@stanford.edu

E. M. Makasa, MBChB, MPH

Counselor-Health: Permanent Mission of Zambia, Geneva, Switzerland

A. W. Gelb, MBChB

Department of Anesthesia \& Perioperative Care, University of California San Francisco, San Francisco, CA, USA some $20-30 \%$ of cases came from direct reports from the ward nurses, undermining the reliability of the mortuary as a single data source for perioperative mortality. Of these 114 deaths, 59 cases had records available for review.

When the authors investigated the preventability of these 59 postoperative deaths, they concluded that 14 were unavoidable and eight were unclear or indeterminate - that is, $60 \%$ of perioperative deaths were considered "avoidable" or "probably avoidable within available resources". Factors contributing to avoidable mortality were surgical (i.e., poor preparation/judgement/technique and undue delays), anesthetic (i.e., poor preparation and postoperative care), and systemic (i.e., lack of available resources, especially blood, intensive care capabilities, and staffing).

The authors also compared their findings with the last published audit from UTH in $1987 .^{2}$ Even under the best possible circumstance (i.e. no deaths in any of the 6,322 cases missing from the theatre logs in the 2012 audit), they found no significant differences in mortality following surgery over the past two decades. Indeed, they noted that perioperative deaths may have actually increased during this time despite global trends showing reductions in surgical mortality. Additionally, preventable deaths appear as common as they were in 1987.

As a first step, let's consider these numbers as a whole. The authors report a $1 \%$ postoperative six-day mortality rate for UTH; whilst from a single centre, this is no worse than national mortality rates for surgery reported by wealthy countries such as the United States (1.14\%) and the Netherlands (1.85\%). ${ }^{3,4}$ At face value, the reported death rate appears acceptable, for despite differences in case mix, these patients generally present with advanced acute illness and significant comorbidities, including malnutrition, anemia (i.e., both chronic as well as 
malaria-related), and immunosuppression (i.e., HIVrelated). Where these mortality figures differ is in the preventable proportions, which lends urgency to a program focused on quality improvement in perioperative care. Fully $50 \%$ of the clearly preventable deaths were deemed to have a surgical contribution, with the major culprit being failure to book the operating theatre in a timely fashion. An additional $30 \%$ of preventable deaths were due to anesthetic contributions, in particular inadequate preparation and poor postoperative care. Finally, 30\% (the number adds to $>100 \%$ due to multiple preventable contributing factors) were due to lack of basic systems of postoperative care that are essential for ensuring the safe recovery of patients undergoing surgery: availability of blood, oxygen, and intensive monitoring such as found in intensive care or postoperative recovery units. Such findings suggest that small but important changes to the processes of care might yield tremendous rewards in terms of surgical safety.

The study did have some notable shortcomings. For example, it was hampered by a lack of standardized data, thus requiring variable contortions to collect, analyze, interpret, and report some of the findings. This limitation serves to highlight that, to date, the collection of perioperative statistics has failed to capture the interest of ministries of health or international bodies, yet it has been fundamental to the improvements in perioperative care realized in high-income countries during the past century. The recommendations for standardized data collection are well established. They include information on how much surgery is performed, the number of providers available for surgical and anesthetic delivery, the infrastructure available for surgery, and the number of deaths following surgery. ${ }^{5}$ In addition, recent work by the Lancet Commission on Global Surgery recommends collecting information on access to care and the costs to patients of such care. ${ }^{6}$ The specifics of such data collection will require refinement with respect to logistics, data sources, and quality assurance mechanisms, but as with other health priorities, such as maternal and neonatal mortality, HIV prevalence, and vaccine coverage, ministries and health systems will compile such information if the international community supports and encourages their efforts.

Collection of such data should not delay the more immediate issues of ensuring routine compliance with basic standards of surgical and anesthetic safety. The WHO led such efforts just six years ago through its support and development of Guidelines for Safe Surgery. ${ }^{7}$ These guidelines were distilled into a Surgical Safety Checklist which has repeatedly been shown to improve outcomes, reduce postoperative deaths, and diminish complications. ${ }^{8-10}$ The World Federation of Societies of Anaesthesiologists has also published International
Standards for the Safe Practice of Anaesthesia and an associated checklist in multiple languages. ${ }^{11,12}$ All these checklists are deceiving, however, as incorporating them into routine care can be surprisingly difficult. Changes to the normal patterns of work are difficult, modification of the operating theatre hierarchy is challenging, and resistance is common. With persistence and collective leadership from hospital administration as well as surgical, nursing, and anesthetic disciplines, long-term and sustainable improvements in practice can be maintained. ${ }^{13}$

The World Health Assembly (WHA), the highest body to set health policy and responsible for governing the World Health Organization (WHO), recently passed resolution WHA68.15, "Strengthening Essential and Emergency Surgery and Anesthesia Care as a Component of Universal Health Coverage". ${ }^{14}$ This is the first global recognition of anesthesia as essential for improving perioperative outcomes and should empower the WHO to push for improvements in these more complex aspects of care delivery. Still, frontline clinicians and health workers need to be supported in their efforts. Ongoing mentoring and training are accepted unequivocally in affluent countries through continuing medical education programs, re-credentialing, and peer-to-peer learning and support. While dedicated programmatic support for such endeavours is lacking in low-resource countries, a number of programs are working to fill this gap. In Zambia, the Tropical Health and Education Trust (THET) has established a training program to educate and develop physician anesthetists through an MMed program. ${ }^{15}$ Twenty-three trainees are currently enrolled, with the first six due to graduate this year and at least eight due to enroll in the 2015/2016 academic year (personal communication: Emily Measures, THET Zambia Country Manager). At present, there are 18 consultant anesthetists in Zambia, all expatriates, so the first cohort alone will result in a $33 \%$ increase. The professionalization of this workforce should create a critical mass of physician providers who can support, encourage, and educate their peers and their junior ranks. Further, we all have much to learn from such colleagues in under-resourced countries who must save lives by astute clinical skill and the ability to improvise.

The effects of ignoring the more complex aspects of care delivery, such as surgery, have come at a high price. While attention has been appropriately focused on population killers such as infectious diseases, malnutrition, and maternal conditions, health capacity and resiliency have generally been neglected. Health systems cannot be improved in sequence; they need to be strengthened in parallel and in concert with multiple programs of care delivery. Strong health systems need forceful clinical advocates, energetic community engagement, and economic vitality. Nevertheless, many 
health programs in resource-constrained settings are often created as a work-around for a poorly functioning health system. Perioperative care cannot be accomplished this way - it is too interdependent on all the components that comprise a strong health system, such as infrastructure, durable and consumable goods, supply chains, human resources, and monitoring and evaluation capabilities. This last element, while seemingly the most dispensable, is in fact essential. Measurement of effect allows systems to understand what has been done, what is working, what is failing, and what is needed. With such knowledge, systems of care can mature, improve outcomes, and provide better care for the populations they serve. This is indeed the effect of measurement - i.e., advances occur when they can be measured and observed over time, thereby allowing the setting up of time-linked improvements in outcomes.

\section{Améliorer les résultats péri-opératoires dans les pays à faibles revenus: pas de résolution sans données}

Mesurer les effets des soins est un principe fondamental de la médecine. Cela inclut non seulement les réponses immédiates aux traitements mais aussi les critères d'évaluation, le plus dramatique et définitif étant la mortalité. La connaissance des taux de décès est essentielle à la compréhension de l'impact des soins péri-opératoires. Cela procure aux cliniciens une orientation indispensable pour identifier les risques et avantages des interventions, aux patients la possibilité de soupeser ces risques, et aux systèmes de soins de santé un moyen d'évaluer la performance, d'apprendre de ceux qui réussissent bien (ou mal) et de poser des jalons en vue d'améliorations.

Dans ce numéro du Journal, Lillie et coll. présentent une enquête sur 18010 patients ayant subi une intervention chirurgicale à l'Hôpital universitaire (UTH) de Lusaka (Zambie) au cours de l'année 2012 - une enquête précédente de ce type avait été publiée 25 ans plus tôt. Les auteurs ont analysé les taux de mortalité postopératoire à 6 jours ainsi que la probabilité que ces décès étaient évitables. ${ }^{1}$ Sur ce total tiré des dossiers administratifs de l'hôpital, les investigateurs ont identifié 11688 cas chirurgicaux à partir des registres de salle d'opération (une perte de $35 \%$ par rapport aux registres administratifs). En croisant les données provenant de la morgue de l'hôpital ainsi que les données des services de chirurgie et d'obstétrique, les auteurs ont pu identifier 114 décès survenus dans les six jours suivant l'intervention chirurgicale. Élément révélateur, entre $20 \%$ à $30 \%$ des cas provenaient directement de rapports des infirmières des services, minant la fiabilité de la morgue comme unique source de données sur la mortalité péri-opératoire. Sur ces 114 décès, il y avait 59 dossiers analysables.

Quand les auteurs ont étudié l'évitabilité de ces 59 décès postopératoires, ils ont conclu que 14 d'entre eux étaient inévitables et que 8 décès avaient une cause indéterminée ou peu claire, ce qui signifiait que $60 \%$ des décès péri-opératoires étaient jugés «évitables » ou «probablement évitables avec les ressources disponibles ». Les facteurs contribuant au caractère évitable de la mortalité étaient en rapport avec la chirurgie (mauvaise préparation/jugement/technique et retards injustifiés, p. ex.), l'anesthésie (mauvaise préparation et soins postopératoires insuffisants, p. ex.) et systémiques (manque de ressources disponibles, notamment de sang, capacités de soins intensifs et manque de personnel, p. ex.).

Les auteurs ont également comparé leurs constatations avec la dernière enquête publiée de l'UTH en $1987 .^{2}$ Même en faisant l'hypothèse la plus favorable possible (c.-à-d. aucun décès parmi les 6322 cas manquants des registres des salles d'opération dans l'enquête de 2012), ils n'ont trouvé aucune différence significative sur la mortalité post chirurgicale depuis les deux dernières décennies. En fait, ils ont noté que les décès péri-opératoires pouvaient avoir, en réalité, augmenté au cours de cette période en dépit des tendances mondiales à la baisse de la mortalité chirurgicale. De plus, les décès évitables semblent être aussi fréquents qu'en 1987.

Considérons tout d'abord ces chiffres dans leur ensemble. Les auteurs indiquent un taux de mortalité postopératoire à 6 jours de $1 \%$ à l'UTH; bien que ce chiffre ne provienne que d'un seul centre, il n'est pas pire que les taux nationaux de mortalité concernant la chirurgie publiés par les pays riches, tels que les États-Unis $(1,14 \%)$ et les Pays-Bas $(1,85 \%){ }^{3,4}$ À première vue, le taux de mortalité publié parait acceptable en dépit des différences dans le mélange des cas, ces patients ayant habituellement une maladie aiguë avancée et des comorbidités significatives, notamment la malnutrition, l'anémie (anémie chronique et anémie liée au paludisme) et une immunosuppression (liée au VIH). La différence dans ces chiffres de mortalité provient des pourcentages de décès évitables, ce qui soulève l'urgente nécessité d'un programme se concentrant sur l'amélioration de la qualité des soins péri-opératoires. Une contribution chirurgicale était attribuée à $50 \%$ des décès manifestement évitables, le principal coupable en étant l'échec de réservation d'une salle d'opération. Le rôle de l'anesthésie était majeur dans $30 \%$ supplémentaires des décès évitables, en raison notamment d'une préparation inadaptée et de soins 
postopératoires insuffisants. Enfin, $30 \%$ des décès évitables (le total est supérieur à $100 \%$ en raison de la multiplicité des facteurs contributifs évitables) étaient dus à l'absence de systèmes de base des soins postopératoires, essentiels pour assurer une récupération sécuritaire des patients ayant subi une chirurgie : la disponibilité de sang, d'oxygène et de monitoring intensif, telle qu'on les trouve dans les unités de soins intensifs ou de soins postopératoires. De telles constatations supposent que des changements limités mais importants apportés aux processus de soins pourraient se traduire par des bénéfices considérables en termes de sécurité chirurgicale.

L'étude présente néanmoins des défauts notables. Elle a été, par exemple, gênée par l'absence de données standardisées, nécessitant donc différentes contorsions pour collecter, analyser, interpréter et rapporter certaines constatations. Cette limitation permet de souligner qu'à ce jour, la collecte des statistiques péri-opératoires n'a pas réussi à capter l'intérêt des ministères de la Santé ou des organismes internationaux; elles ont pourtant joué un rôle fondamental dans l'amélioration des soins péri-opératoires qui a eu lieu dans les pays à hauts revenus au cours du dernier siècle. Les recommandations pour la collecte de données standardisées sont maintenant bien connues. Elles incluent de l'information sur le nombre d'interventions pratiquées, le nombre de praticiens disponibles pour assurer l'anesthésie et la chirurgie, l'infrastructure chirurgicale disponible et le nombre de décès après l'intervention chirurgicale. ${ }^{5}$ De plus, une étude récente de la Lancet Commission on Global Surgery recommande de collecter de l'information sur l'accès aux soins et le coût de ces soins pour les patients. ${ }^{6}$ La spécificité de la collecte de ces données va nécessiter un raffinement de la logistique, des sources de données et des mécanismes d'assurance de la qualité, mais comme pour d'autres priorités sanitaires, telles que la mortalité maternelle et néonatale, la prévalence du VIH, la couverture vaccinale, les ministères et les systèmes de santé compileront de telles informations si la communauté internationale soutient et encourage leurs efforts.

La collecte de ces données ne doit pas retarder le problème plus immédiat qu'est le respect quotidien des normes de base de la sécurité chirurgicale et anesthésique. L'OMS a mené de tels efforts il y a juste six ans via son soutien et le développement de lignes directrices pour une chirurgie sécuritaire (Guidelines for Safe Surgery). ${ }^{7}$ Ces lignes directrices ont été résumées dans une liste de contrôle de la chirurgie sécuritaire (Surgical Safety Checklist) qui a régulièrement prouvé qu'elle améliorait les résultats, réduisait le nombre de décès postopératoires ainsi que les complications. ${ }^{8-10}$ La Fédération internationale des sociétés d'anesthésiologistes a également publié des normes internationales pour la pratique sécuritaire de l'anesthésie
(International Standards for the Safe Practice of Anaesthesia) et une liste de contrôle associée dans différentes langues. ${ }^{11,12}$ Toutes ces listes de contrôle sont toutefois trompeuses, car leur inclusion dans la pratique de soins quotidienne peut s'avérer étonnamment difficile. Il est difficile d'obtenir des modifications des habitudes de travail et de la hiérarchie en salle d'opération, et les résistances sont courantes. Avec de la persévérance et le leadership collectif de l'administration hospitalière,et des disciplines de chirurgie, de soins infirmiers et d'anesthésiologie, des améliorations durables et à long terme des pratiques peuvent être maintenues. ${ }^{13}$

L'Assemblée mondiale de la santé (AMS), l'organisation la plus haut placée pour l'établissement des politiques et responsable du fonctionnement de l'Organisation mondiale de la santé (OMS), a récemment passé la résolution WHA68,15, « renforçant la place des soins chirurgicaux et anesthésiques essentiels et urgents comme éléments de la couverture de santé universelle ». ${ }^{14} \mathrm{Il}$ s'agit de la première reconnaissance internationale du caractère essentiel de l'anesthésie pour l'amélioration des résultats péri-opératoires et elle doit donner à l'OMS la force d'insister sur les améliorations à apporter à ces aspects plus complexes des soins. Néanmoins, les efforts des cliniciens et professionnels de la santé qui sont en première ligne doivent être soutenus. Le mentorat et la formation continue sont admis de façon non équivoque dans les pays développés par le biais de programmes de formation médicale continue, de réévaluation des compétences et d'apprentissage et de soutien direct entre collègues. Bien qu'un soutien programmatique dédié à de telles entreprises fait défaut dans les pays à faibles ressources, un certain nombre de programmes s'efforcent de combler ce manque. En Zambie, le Tropical Health and Education Trust (THET) a mis en place un programme de formation MMed pour l'éducation et la progression des médecins anesthésiologistes. ${ }^{15}$ Vingt-trois stagiaires sont actuellement inscrits et les six premiers seront diplômés cette année; au moins huit autres doivent s'inscrire pour l'année universitaire 2015/2016 (communication personnelle : Emily Measures, directrice nationale de THET Zambie). À l'heure actuelle, il y a 18 anesthésiologistes en Zambie, tous expatriés, si bien que cette première cohorte entraînera à elle seule une augmentation de leur nombre de $33 \%$. La professionnalisation de cet effectif doit créer une masse critique de fournisseurs de soins de santé capables de soutenir, encourager et éduquer leurs pairs et collègues plus jeunes. En outre, nous avons beaucoup à apprendre de tels collègues des pays en manque de ressources, car ils doivent sauver des vies en faisant preuve de compétences cliniques, d'astuce et de capacités d'improvisation.

Ignorer les aspects plus complexes de la délivrance de soins, tels que la chirurgie, a un prix élevé. Alors que 
l'attention s'est portée avec logique sur les tueurs de populations, comme les maladies infectieuses, la malnutrition et les maladies maternelles, les moyens sanitaires et la résilience ont été souvent négligés. Les systèmes de santé ne peuvent pas être améliorés par à-coups; ils doivent être renforcés parallèlement et de conserve avec de multiples programmes de fournitures de soins. Des systèmes de santé forts nécessitent des porte-parole cliniques énergiques, un solide engagement communautaire et une vitalité économique. Cependant, de nombreux programmes de santé dans les cadres aux ressources limitées sont créés en tant que solution de dépannage dans un système sanitaire peu fonctionnel. Les soins péri-opératoires ne peuvent pas être menés de cette façon: ils sont trop interdépendants de toutes les composantes qui constituent un système de soins fort, comme son infrastructure, des produits durables et des consommables, des chaînes d'approvisionnement, des ressources humaines ainsi que des capacités de contrôle et d'évaluation. Ce dernier élément, bien que paraissant le moins indispensable, est en fait essentiel. La mesure de l'effet permet aux systèmes de comprendre ce qui a été fait, ce qui fonctionne, ce qui échoue et ce qui est nécessaire. Avec ces connaissances, les systèmes de soins peuvent progresser, améliorer les résultats et apporter de meilleurs soins à leurs populations. C'est incontestablement l'effet de la mesure : il y a progrès quand ils peuvent être mesurés et observés au fil du temps, permettant d'établir des échéances pour les améliorations à venir dans les résultats.

Conflicts of interest None declared.

Disclaimer The views expressed here are those of the authors and do not necessarily represent the views of the Government of Zambia or the World Federation of Societies of Anaesthesiologists.

Conflits d'intérêts Aucun déclaré.

Clause de non-responsabilité Les points de vue exprimés ici sont ceux des auteurs et ne représentent pas nécessairement les points de vue du gouvernement de la Zambie ou de la Fédération internationale des sociétés d'anesthésiologistes.

\section{References}

1. Lillie EM, Holmes CJ, O'Donohoe EA, et al. Avoidable perioperative mortality at the University Teaching Hospital, Lusaka, Zambia: a retrospective cohort study. Can J Anesth 2015; 62: this issue. DOI:10.1007/s12630-015-0483-z.

2. Heywood AJ, Wilson IH, Sinclair JR. Perioperative mortality in Zambia. Ann R Coll Surg Engl 1989; 71: 354-8.

3. Weiser TG, Semel ME, Simon AE, et al. In-hospital death following inpatient surgical procedures in the United States, 1996-2006. World J Surg 2011; 35: 1950-6.

4. Noordzij PG, Poldermans D, Schouten O, Bax JJ, Schreiner FA, Boersma D. Postoperative mortality in The Netherlands: a population-based analysis of surgery-specific risk in adults. Anesthesiology 2010; 112: 1105-15.

5. Weiser TG, Makary MA, Haynes AB, et al. Standardised metrics for global surgical surveillance. Lancet 2009; 374: 1113-7.

6. Meara JG, Leather AJ, Hagander L, et al. Global surgery 2030: evidence and solutions for achieving health, welfare, and economic development. Lancet 2015; . DOI:10.1016/S01406736(15)60160-X.

7. World Health Organization. WHO Guidelines for Safe Surgery. Geneva, 2009. Available from URL: http://whqlibdoc.who.int/ publications/2009/9789241598552_eng.pdf (accessed July 2015).

8. Haynes $A B$, Weiser $T G$, Berry WR, et al. A surgical safety checklist to reduce morbidity and mortality in a global population. N Engl J Med 2009; 360: 491-9.

9. de Vries EN, Prins HA, Crolla RM, et al. Effect of a comprehensive surgical safety system on patient outcomes. N Engl J Med 2010; 363: 1928-37.

10. van Klei WA, Hoff RG, van Aarnhem EE, et al. Effects of the introduction of the WHO "Surgical Safety Checklist" on inhospital mortality: a cohort study. Ann Surg 20102; 255: 44-9.

11. World Federation of Societies of Anaesthesiologists. Safety and Quality. Available from URL: http://www.wfsahq.org/our-work/ safety-quality (accessed July 2015).

12. Merry AF, Cooper JB, Soyannwo O, Wilson IH, Eichhorn JH. International standards for a safe practice of anesthesia. Can J Anesth 2010; 57: 1027-34.

13. Kim RY, Kwakye G, Kwok AC, et al. Sustainability and long-term effectiveness of the WHO Surgical Safety Checklist combined with pulse oximetry in a resource-limited setting: two-year update from Moldova. JAMA Surg 2015; 150: 473-9.

14. World Health Organization. Sixty-Eighth World Health Assembly. Available from URL: http://www.who.int/ mediacentre/events/2015/wha68/en/ (accessed July 2015).

15. Kinnear JA, Bould MD, Ismailova F, Measures E. A new partnership for anesthesia training in Zambia: reflections on the first year. Can J Anesth 2013; 60: 484-91. 